\title{
Calidad del agua de la parte baja del río Córdoba (Magdalena, Colombia), usando el ICA-NSF
}

\section{Water quality in the lower section of Córdoba River (Magdalena, Colombia), using the ICA-NSF}

\author{
Fernando Antonio Fontalvo-Julio y Cesar E. Tamaris-Turizo@1*
}

*Autor de correspondencia: ctamaris@unimagdalena.edu.co

Recibido: 18 abril de 2018 - Aceptado: 23 de agosto de 2018

1. Grupo de investigación de Biodiversidad y Ecología Aplicada, Universidad del Magdalena, Santa Marta, Colombia

\section{Resumen}

Palabras clave:

parámetros fisicoquímicos; índice de calidad del agua; recurso hídrico; río Neotropical

Key words:

parameter physicochemical; water quality index; water resource; Neotropical river
La evaluación de la calidad del agua de fuentes fluviales a través del análisis de variables físicas, químicas y microbiológicas es considerada como una herramienta que permite conocer su estado de contaminación. Una de las formas de evaluar estos sistemas es a través de la integración de variables ambientales mediante índices como el ICA-NSF. Por tanto, el objetivo de este trabajo fue evaluar la calidad fisicoquímica de las aguas del río Córdoba en su parte baja a través del índice ICA-NSF y analizar los plaguicidas organoclorados más comunes. Se definieron seis sitios de muestreo, en cada uno de estos se midieron parámetros físicos, químicos y microbiológicos. Además, se determinó el nivel de concentración de los principales contaminantes, considerando el impacto que causan en el río y se estableció la calidad del agua durante los periodos de sequías y lluvias entre el 2010 y el 2011. El ICA-NSF varió entre 52,6 durante las lluvias en "Muelle carbonífero" y 72,4 en el periodo seco en "Paso de los anillos". Lo anterior indica que la calidad del agua categorizó entre regular y buena, respectivamente. De las variables evaluadas, los coliformes fecales mostraron altos valores en los seis sitios (entre 2974 y $4940 \mathrm{NMP} / 100 \mathrm{~mL}$ ), registros que sobrepasaron los límites permisibles para el consumo humano y la protección de flora y la fauna por la legislación colombiana. Los resultados mostraron una variación temporal (entre periodos climáticos) y una alta uniformidad espacial del río en los sitios de muestreo. En ninguno de los muestreos se encontraron residuos de plaguicidas organoclorados. Los resultados evidenciaron que la variación temporal (periodos de lluvias vs. sequías) reflejó de mejor manera los cambios en la calidad del agua del río Córdoba en su parte baja.

\section{Abstract}

The evaluation of the water quality of fluvial sources through the analysis of physical, chemical and microbiological variables is considered as a tool that allows to know the status of contamination. One of the forms to evaluate these systems is through the integration of environmental variables in indexes as the ICA-NSF. Therefore, the objective of this study was to evaluate the physicochemical quality of the lower part of the Córdoba River through the ICA-NSF index and analyze the most common pesticides. Six sampling stations were defined, in each station, physical, chemical and microbiological parameters were measured. In addition, the level of concentration of the main pollutants was determined, considering the impact they cause on the river and the water quality was established through the water quality index (ICA-NSF) during the dry and rainy periods between the 2010 and 2011. The ICA-NSF varied between 52.6 during the rains in the "Muelle carbonifero" and 72.4 in the dry period in "Paso de los anillos". That indicated that the water quality was classified between regular and good, respectively. From the evaluated variables, the fecal coliforms showed high values in the six stations (between 2974 and 4940 NMP / $100 \mathrm{~mL}$ ), records that exceed the limits accepted for human consumption and the protection of flora and fauna by the Colombian legislation. The results showed a temporal variation (between climatic periods) and a high spatial uniformity. Any samples showed presence of organochlorine pesticides. The results showed that the temporal variation (rainy vs. dry seasons) was the best descriptor to reflect the water quality of the lower part of the Cordoba River. 


\section{Introducción}

El agua es patrimonio de la humanidad, como elemento de la naturaleza y como recurso indispensable para las actividades económico-productivas (Parra, 2009). El uso y manejo de las fuentes hídricas es un reto para las instituciones, las autoridades, los grupos sociales y demás entes responsables de la conservación de la calidad del agua en diversos sistemas naturales. Por esto es importante conocer las dinámicas de las variables ambientales que pueden cambiar las condiciones normales del sistema, como es el caso de la calidad física y química que pueden afectar a la salud (OMS, 2006; Díaz et al., 2018). Desde el punto de vista microbiológico, la calidad del agua en corrientes naturales y redes de distribución puede variar rápidamente en el espacio y el tiempo (Torres et al., 2008). Sin embargo, una significativa alteración de la microbiología del sistema puede impactar negativamente la biota y la calidad sanitaria del agua (Camacho et al., 2009; Castro et al., 2014). Los coliformes son abundantes y casi exclusivos de la materia fecal, las características de sobrevivencia y la capacidad para multiplicarse fuera del intestino también se observan en aguas potables, por lo que se utiliza comúnmente como indicador de contaminación fecal en aguas; conforme mayor sea el número de coliformes en agua, mayor será la probabilidad de estar frente a una contaminación reciente (Camacho et al., 2009).

La composición química del agua de un río es determinada por varios factores como la precipitación, la geología, la solubilidad de las rocas o materiales, la vegetación terrestre, los procesos de evaporación, los procesos biológicos y la contaminación o vertidos de origen humano que se dan en la cuenca (Toro et al., 2002). Por tanto, la evaluación de la calidad del agua usualmente se realiza usando técnicas analíticas adecuadas para cada caso. De esta manera, los resultados de estas determinaciones serán representativos (Barrenechea, 2004; Torres et al., 2009). Con el fin de facilitar la interpretación de los datos químicos, físicos y biológicos, cada vez más agencias medioambientales, universidades e institutos recurren a los índices de calidad y contaminación del agua (ICA e ICO), los cuales mediante expresión matemática, que representa todos los parámetros valorados, permiten evaluar el recurso hídrico (Ball y Church, 1980; SamboniRuíz et al., 2007). En Colombia se han realizado algunos trabajos que han aplicado el ICA en ríos andinos. De ellos se destacan los de Behar et al. (1997), Jiménez y Vélez (2006), Montoya et al. (2011), Forero-Céspedes et al. (2013), Bustamante et al. (2014) y Valverde-Solís et 102 al. (2015). En el departamento del Magdalena, solo se registra un trabajo en el que evalúan la calidad física y química del agua en ríos como el Manzanares (Romero et al., 2011).

La calidad de agua puede ser usada para realizar un diagnóstico, toda vez que se trata de un proceso de evaluación total de la naturaleza física, química y biológica; en relación a la condición natural, a los efectos humanos y a los usos intencionales, particularmente los que puedan afectar la salud humana, y a la de los sistemas acuáticos (Chapman, 1996). La evaluación de la calidad en los recursos hídricos, usualmente se realiza a través de estudios que incorporan variables espaciales y temporales, la primera variable suele hacerse a través de un gradiente altitudinal o de cambios considerables graduales en un ecosistema, mediante selección un área representativa del objeto de estudio. Mientras que en la evaluación temporal en ecosistemas tropicales resulta indispensable el contraste de los periodos climáticos, debido a que contribuyen de manera significativa a la estructura biológica y ambiental de los ecosistemas, lo cual afecta la interpretación de los resultados que se puedan obtener (Roldán y Ramírez, 2008; Zúñiga y Cardona, 2009).

La utilización de los plaguicidas para combatir las enfermedades en plantas incluye compuestos organoclorados, como el DDT y el aldrin cuya acción puede tardar cerca de veinte años. En el agua se detecta como componente de contaminación, poco usado en gran parte del mundo. Durante la Convención de Estocolmo sobre Contaminantes Orgánicos Persistentes (COP’s) en mayo de 2001, se aprobó su eliminación de dichos componentes. En principio están identificados como COP's los plaguicidas DDT, aldrin, dieldrin, endrin, heptacloro, metoxicloro, toxafeno, endosulfan y el hexaclorobenceno (BHC) y otros compuestos clorados como los bifenilos policlorados (PCBs), las dioxinas y los furanos. En Colombia se prohibió el uso y venta de insecticidas organoclorados en el cultivo del tabaco y del café por medio de la Resolución 447 del 6 de diciembre de 1974 y la de 209 del 12 de mayo de 1978 respectivamente, expedidas por el Ministerio de Agricultura.

El objetivo de este trabajo fue evaluar la calidad del agua en la parte baja del río Córdoba, a partir del análisis de variables físicas y químicas, y microbiológicas (coliformes totales y fecales), además de la integración del índice de calidad de agua ICA-NSF. Se espera que el agua refleje una mejor calidad durante los periodos lluviosos, debido a que las precipitaciones actúan como diluyentes de los contaminantes. En su lugar, durante los periodos de sequía la reducción del caudal favorece la concentración de dichos 
contaminantes y con ello el reflejo de peores condiciones de calidad de agua.

\section{Materiales y métodos}

\section{Área de estudio}

La cuenca del río Córdoba tiene un área de $125 \mathrm{~km}^{2}$ (ProSierra, 1991). Nace en el sector occidental de la Serranía de San Lorenzo de la Sierra Nevada de Santa Marta (SNSM) a una altitud aproximada de $1900 \mathrm{~m}$. El río fluye en su curso alto y medio por un estrecho valle en forma de "V". En su parte baja avanza por una estrecha faja de tierra unos $4 \mathrm{~km}$ hasta desembocar en el mar Caribe (sector La Punta de Córdoba) a 2,5 km al norte del municipio de Ciénaga (Cadavid, 1985). La temperatura promedio mensual multianual es de $28{ }^{\circ} \mathrm{C}$ (Minambiente, 2005); el caudal promedio mínimo en marzo fue de $1,60 \mathrm{~m}^{3} / \mathrm{s}$ y de 12,53 $\mathrm{m}^{3} / \mathrm{s}$ en octubre. Durante el 2009 la precipitación promedio multianual del municipio de Ciénaga alcanzó los 731,3 mm. El clima de la cuenca se caracteriza por tener déficit de agua durante casi tres meses del año (CORFAS, 1990).

La cuenca del río Córdoba es considerado como una de las fuentes hídricas más importantes del departamento del Magdalena, debido a que sus aguas son utilizadas para la agricultura, la pesca, el turismo y el consumo de los habitantes del municipio de Ciénaga y otras poblaciones aledañas (CODECI, 1996). Actividades como la agricultura y el turismo generan constantemente vertimientos de residuos agroquímicos (fertilizantes y plaguicidas), grasas, aceites, detergentes, residuos vegetales, excreciones de animales y humanas que llegan al río por varias vías de escorrentía (CODECI, 1996), las cuales afectan de manera negativa la calidad del agua del río.

\section{Recolección y procesamiento de muestras}

En cada uno de los seis sitios de muestreo se realizaron cuatro campañas, cubriendo los periodos de sequía $(11 / 08 / 2011$ y $20 / 03 / 2012)$ y lluvias $(10 / 12 / 2010$ y $10 / 11 / 2011)$. Todos los sitios se georreferenciaron, describieron y nominaron de acuerdo a su localización (tabla 1). En cada lugar se seleccionó un tramo recto de aproximadamente $100 \mathrm{~m}$ con flujo de agua uniforme donde se midieron algunas variables físicas y químicas como el $\mathrm{pH}$, la conductividad, el oxígeno disuelto y la temperatura empleando un equipo multiparámetro WTW 350i/set 2F40114BOE. Los caudales se midieron con la ayuda de un objeto flotador (Hudson, 1997). Adicionalmente, en cada sitio se tomaron tres muestras de agua que se refrigeraron en campo a $4^{\circ} \mathrm{C}$ y se transportaron al laboratorio de Calidad de Aguas de la Universidad del Magdalena para el análisis de las variables físicas y químicas.

La determinación de plaguicidas organoclorados del agua se realizó en un cromatógrafo de gases (GC) HP 6890 Series PLUS, acoplado a un detector de captura de electrones (ECD). La columna empleada en el análisis DB-5 $5 \%$ - fenol - poli (metilsiloxano), $30 \mathrm{~m}$ x 0,25 $\mathrm{nm}$ x 0,25 $\mu \mathrm{m}$. La inyección se realizó en modo splitless mediante la técnica de extracción sólido (líquido con hexano) acetona para sedimentos, y extracción sólido (líquido con acetonitrilo a temperatura ambiente y agitación). La determinación de coliformes totales y fecales se realizó usando el método estándar de NMP en tubos múltiples (APHA, 1998). La estimación de los nitratos se realizó por el método de reducción con Cd$\mathrm{Cu}$ /colorimetría. Los nitritos se evaluaron con la aplicación de Sulfanilamida/colorimetría y la evaluación de fósforo se aplicó al método del ácido ascórbico (Murphy y Riley, 1962).

Se calculó el Índice de Calidad del Agua (ICA) de la National Sanation Fundation - NSF de los Estados Unidos, mediante la asignación de pesos específicos definidos por Brown et al. (1970) y empleando la función ponderada multiplicativa (ICAm) como sigue:

$$
I C A_{m}=\prod_{i=l}^{9}\left(S u b_{i}^{w_{i}}\right)
$$

Dónde: wi: peso asignado a cada parámetro ponderados entre 0 y 1 . Subi: subíndice del parámetro i. Los Subi se hallaron utilizando las curvas de funcionamiento para los parámetros establecidos de acuerdo al procedimiento diseñado por Brown et al. (1970). Las variables seleccionadas para el ICA fueron: coliformes fecales, pH, DBO5 nitratos, fosfatos, temperatura, turbidez, sólidos disueltos y oxígeno disuelto. Este índice considera las categorías de calidad de las aguas con su correspondiente puntuación así: Muy Mala con valores entre 0 y 25; Mala entre 26 y 50; Media entre 51 y 70; Buena entre 71 y 90 y Excelente entre 91 y 100.

\section{Análisis de datos}

Se realizaron gráficos de dispersión para conocer la tendencia de las variables ambientales a lo largo de los sitios por cada muestreo. Se calculó el ICA-NSF siguiendo las recomendaciones de Brown et al. (1970), para conocer la calidad del agua en los sitios y durante los muestreos. Finalmente, se realizó un análisis de escalamiento espacial nMDS entre las fechas de muestreo para conocer la asociación de las variables ambientales durante los periodos climáticos (sequía y lluvias). Este análisis se realizó en el programa R (R Development Core Team, 2002). 
Tabla 1. Descripción de los sitios de muestreo de la parte baja del río Córdoba.

\begin{tabular}{clrrl} 
Sitio & Nombre del sitio & $\begin{array}{r}\text { Altitud } \\
(\mathrm{m})\end{array}$ & $\begin{array}{r}\text { Coordenadas } \\
(\text { UTM) }\end{array}$ & Descripción del sitio \\
\hline $\begin{array}{l}\text { Paso de los } \\
\text { anillos }\end{array}$ & 40 & $11^{\circ} 01^{\prime} 22,99^{\prime \prime} \mathrm{N}$ & $\begin{array}{l}\text { Se observó el vertimiento de agroquímicos por escorrentía. Es } \\
\text { frecuentado por residentes del sector, animales domésticos, y } \\
\text { por turistas durante los fines de semana. }\end{array}$
\end{tabular}

Hay cultivos de hortalizas y frutales, principalmente: limón, mango, guayaba zapote, yuca y maíz. Hay un alto número de

2 Puente Córdoba $27 \quad \begin{array}{r}11^{\circ} 01^{\prime} 46,5^{\prime} \mathrm{N} \\ 74^{\circ} 12^{\prime} 14,4 \text { ” } \mathrm{W}\end{array}$
residentes en la ribera. Se observaron vertimientos de desechos de construcción. En el sitio frecuentaban animales domésticos y silvestres. También se vertían agroquímicos por vías de escorrentía.

Presentó una vegetación ribereña fragmentada dominada por arbustos y plantas pequeñas. Se observó vertimiento de aguas

3 Finca la India

20

$11^{\circ} 02^{\prime} 04,8^{\prime \prime} \mathrm{N}$ $74^{\circ} 13^{\prime} 08,5^{\prime \prime} \mathrm{W}$

$11^{\circ} 02^{\prime} 11,4 ” \mathrm{~N}$ $74^{\circ} 14^{\prime} 17,5^{\prime \prime} \mathrm{W}$

$4 \quad$ Finca el Confite

10

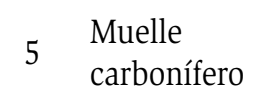
$7 \quad \begin{array}{r}11^{\circ} 02 ' 23,3^{\prime} \mathrm{N} \\ 74^{\circ} 14^{\prime} 23,9^{\prime} \mathrm{W}\end{array}$

6 Desembocadura de riego. El lecho del río presentó material rocoso y sedimentos que son intensamente removidos y extraídos con fines de construcción.

Predominaron plantaciones de banano en las que se aplicaban agroquímicos. El lecho del río presentó material rocoso y sedimentos. Se observaron frecuentes vertimientos domésticos provenientes de lavados y baños de agricultores y visitantes.

El lavado de ropa, animales y vehículos se observó en el sitio; además, vertimiento de agroquímicos. El baño de trabajadores de fincas y del muelle carbonero estaba instalado cerca del sitio de muestreo.

Es un sector muy cercano a la desembocadura del río, el material rocoso es menor que los encontrados en los otros sectores, y son notorios los residuos vegetales y animales contenidos en el sistema hídrico.

\section{Resultados}

\section{Variables físicas, químicas y microbiológicas}

El sitio uno presentó el mayor caudal $\left(14 \mathrm{~m}^{3} / \mathrm{s}\right)$ durante los periodos lluviosos, mientras que en los periodos de sequía el caudal promedio fue de $3,1 \mathrm{~m}^{3} / \mathrm{s}$. Se observaron valores muy altos en la DBO durante el primer periodo lluvioso $(10,75$ - 17,56 mg/L), en comparación con el 104 segundo periodo $(3,12 \mathrm{mg} / \mathrm{L}-9,88 \mathrm{mg} / \mathrm{L})$, tendencia similar a la encontrada durante los periodos de sequía (figura 1a). La mayor variación de los registros del oxígeno disuelto en el agua se observó en los periodos de lluvias, los cuales estuvieron entre 5,5 y $8,2 \mathrm{mg} / \mathrm{L}$, mientras en los periodos secos fue menor $(4,11$ y $6,51 \mathrm{mg} / \mathrm{L}$ ) (figura $1 \mathrm{~b}$ ). Los valores de $\mathrm{pH}$ fueron más altos en el primer periodo lluvioso (figura 1c). Mientras que la temperatura mostró una tendencia espacial, de tal manera que los sitios uno, dos y tres tuvieron mayor variabilidad; por su parte, los sitios cuatro, cinco y seis fueron muy similares (figura 1d). 

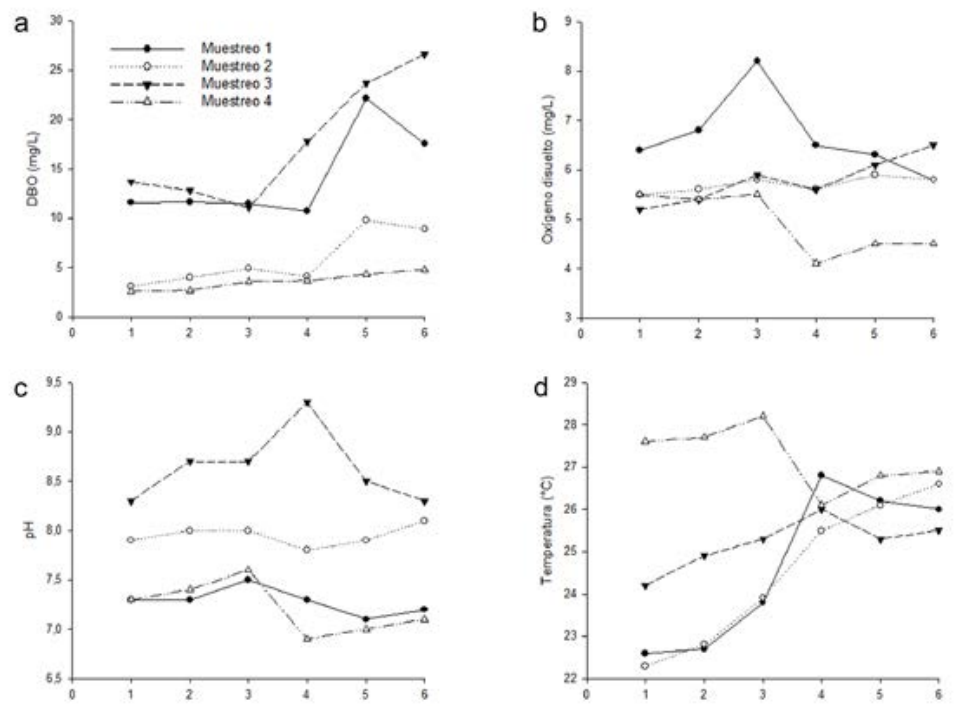

Figura 1. Variación espacial y temporal de: a) la DBO, b) el oxígeno disuelto, c) el pH y d) la temperatura en el río Córdoba. Los números indican los sitios de muestreo relacionados en la tabla 1.

Con relación a los nutrientes, en el sitio seis se presentaron los valores más altos de nitratos $(0,90 \mathrm{mg} / \mathrm{L})$ en el primer periodo lluvioso, y el más bajo $(0,39 \mathrm{mg} / \mathrm{L})$ en el segundo periodo seco (figura 2a). En cuanto a los nitritos, en el primer periodo lluvioso se encontró un rango de 0,05 a $0,11 \mathrm{mg} / \mathrm{L} \mathrm{NO}_{2}$, el segundo periodo seco presentó poca variación $\left(0,12\right.$ y $\left.0,23 \mathrm{mg} / \mathrm{L} \mathrm{NO}_{2}\right)$, el segundo periodo lluvioso estuvo entre $0,08-0,17 \mathrm{mg} / \mathrm{L} \mathrm{NO}_{2}$ y el segundo periodo seco mostró la mayor variación (de 0,009 a 0,21 $\mathrm{mg} / \mathrm{L} \mathrm{NO}_{2}$ ). Los valores de coliformes totales (figura $2 \mathrm{~b}$ ) mostraron que el primer periodo lluvioso comprendió valores entre 855 - $4750 \mathrm{NMP} / 100 \mathrm{~mL}$ y el segundo entre 845 - $4560 \mathrm{NMP} / 100 \mathrm{~mL}$. El primer periodo osciló entre 3895 - $5035 \mathrm{NMP} / 100 \mathrm{~mL}$ y en el segundo resultado estuvo entre 4028 - $8398 \mathrm{NMP} / 100 \mathrm{~mL}$ (figura 2c y 2d). El análisis de plaguicidas no registró niveles detectables de organoclorados por encima del nivel mínimo del método empleado $(<0,1 \mathrm{mg} / \mathrm{L})$ que incluyen los siguientes: lindano, heptacloro, aldrín, heptacloro epóxido, trans clordano, endosulfan I, cis-clordano, dieldrin, 4,4- DDE, endrin, endosulfan II, 4,4DDD, Endosulfan sulfato, 4,4 DDT y endrin cetona (anexo 1).
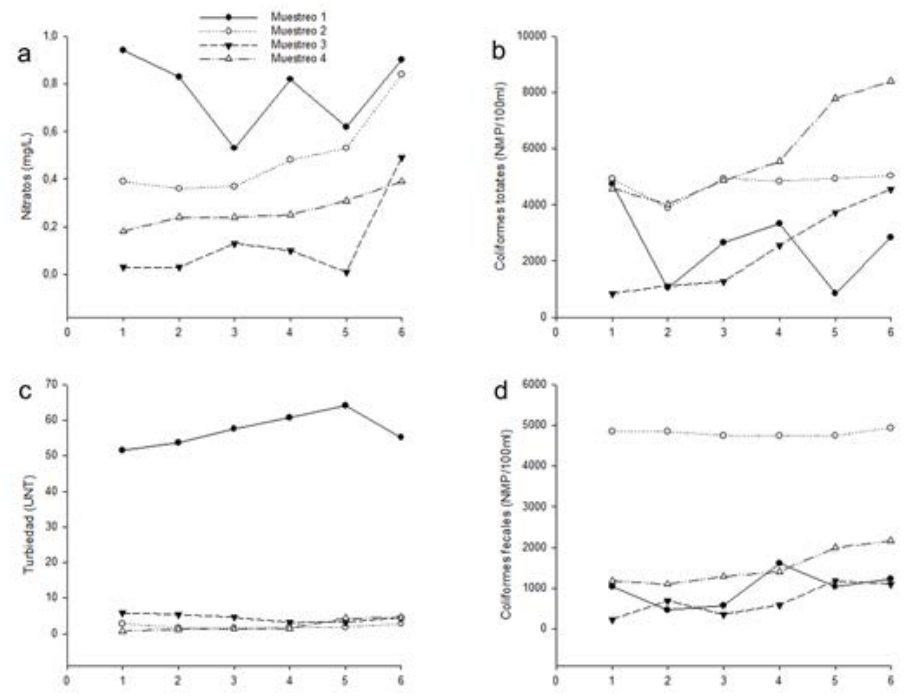

Figura 2. Variación espacial y temporal de: a) los nitratos, b) los coliformes totales, c) la turbiedad y d) los coliformes fecales en el río Córdoba. Los números indican los sitios de muestreo relacionados en la tabla 1 
El análisis nMDS mostró que las variables ambientales valoradas en la parte baja del río Córdoba registraron un comportamiento configurado por los periodos climáticos y no por la ubicación de los sitios (figura 3). Sin embargo, cada muestreo se agrupó de manera independiente, esto indica que las condiciones ambientales durante cada muestreo fueron particulares y que el segundo periodo de sequía presentó los mayores valores en los nutrientes y coliformes, hecho que lo ubicó en el nMDS distante de los demás muestreos.

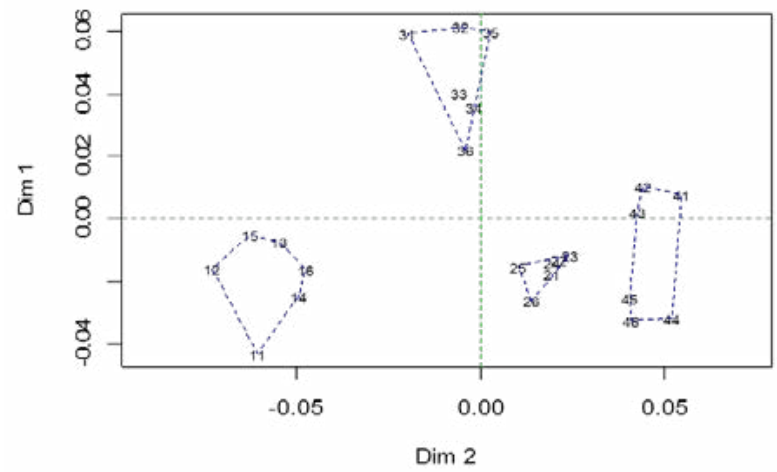

Figura 3. Análisis de ordenación por nMDS de los sitios de estudio, de acuerdo a los periodos climáticos muestreados en la parte baja del río Córdoba.

\section{ICA-NSF}

La calidad del agua en todos los sitios, durante ambos periodos climáticos presentó un ICA-NSF categorizado como Regular, con excepción de los sitios del uno al cuatro durante el muestreo realizado el 20 de marzo de 2012, donde la calidad del agua se categorizó como Buena. Los valores del ICA-NSF fueron mayores durante el periodo de sequía, los cuales oscilaron entre 60,3 y 72,4 , mientras que durante las lluvias los valores estuvieron entre 52,6 y 63,6. Los mayores valores del ICA-NSF en ambos periodos climáticos se observaron en el sitio uno (Paso de los anillos) y los menores en el sitio cinco (Muelle carbonífero) (tabla 2).

\section{Discusión}

Los resultados de este trabajo evidencian que no hay importantes variaciones en las condiciones de la calidad del agua (valoradas a través del ICA-NSF) al comparar las variables físicas y químicas entre los sitios, pero sí entre los periodos climáticos. Esto indica que los cambios en los descriptores ambientales del río fueron promovidos fundamentalmente por la influencia de la variabilidad climática y no por las actividades antrópicas que se realizaban a lo largo del tramo evaluado, debido a la capacidad depuradora del sistema.

Invemar Redcam (2003) registró valores de pH entre 6,5 y 7,50 en sistemas fluviales del departamento del Magdalena. Los valores de $\mathrm{pH}$ observados en el río fueron cercanos al neutral y en algunos casos ligeramente básicos $\approx 9$ unidades. Dichos valores exceden el máximo valor establecido en el decreto 1594 de 1984 actualizado por la resolución 2115 de 2007 del decreto 1575 para consumo humano. Estos resultados probablemente se relacionan con actividades de intensa remoción de material calcáreo que se realiza en el tramo medio del río que puede ser arrastrado por las lluvias. Los coliformes fecales presentados en las cuatro mediciones indican que en todas los sitios, los valores superan los límites establecidos por la legislación colombiana para consumo humano y lo fijado por la CE- CCA/89 para vida acuática (200 NMP/100 mL). Sin embargo, los datos son muy similares a los registrados en la parte baja del río Manzanares en el departamento del Magdalena (Romero et al., 2011) y en los ríos Meléndez y Cali en el departamento del Valle del Cauca (Behar et al., 1997).

Los bajos valores de oxígeno disuelto detectados en los sitios cinco y seis durante los dos primeros muestreos, se relacionan con el alto valor de DBO en dichos lugares. Estos sitios están localizados en un sector cercano a la desembocadura del río, donde se vierten aguas residuales producto del lavado de ropa y vehículos. A pesar de esto, los valores de DBO se consideran aceptables, con indicio de contaminación (Pacheco, 2006). El lavado de ropa y vehículos y los vertimientos de aguas residuales que se realizaba en los sitios del estudio contribuyen al aumento del contenido de materia orgánica en este sector del río, lo que a su vez disminuye la cantidad de oxígeno disuelto en el agua, necesario para la oxidación bioquímica aeróbica de las sustancias orgánicas presentes en el agua (Barón et al., 2003; Rivera et al., 2004; Rúa, 2015). Los valores de nitratos y hierro más altos (tabla 3) que se observaron durante el primer periodo lluvioso, en el sitio uno, pueden obedecer a la mineralización natural del cuerpo de agua y a la geología de la cuenca. Por otro lado, los valores de turbiedad en el primer y el tercer muestreo, fueron mayores al límite establecido por la legislación colombiana para consumo humano (2 NTU). Estos valores en periodo lluvioso se deben principalmente a la cantidad de sólidos en suspensión, producto del arrastre de materia viva y muerta de los sentamientos en los alrededores de los ríos (Caballero, 2007; Romero et al., 2011). 
Tabla 2. Valores y su respectiva categoría del ICA calculado en la parte baja del río Córdoba.

\begin{tabular}{|c|c|c|c|c|c|}
\hline Fecha & Periodo & Sitio & Valor ICA - NSF & Categoría & Color \\
\hline \multirow[t]{6}{*}{$10 / 12 / 2010$} & Lluvias & Paso de los anillos & 54,6 & Regular & \\
\hline & & Puente Córdoba & 57,7 & Regular & \\
\hline & & Finca la India & 57,0 & Regular & \\
\hline & & Finca el Confite & 54,5 & Regular & \\
\hline & & Muelle carbonífero & 52,6 & Regular & \\
\hline & & Desembocadura & 53,2 & Regular & \\
\hline \multirow[t]{6}{*}{$11 / 08 / 2011$} & Sequía & Paso de los anillos & 66,1 & Regular & \\
\hline & & Puente Córdoba & 65,1 & Regular & \\
\hline & & Finca la India & 64,8 & Regular & \\
\hline & & Finca el Confite & 65,2 & Regular & \\
\hline & & Muelle carbonífero & 60,3 & Regular & \\
\hline & & Desembocadura & 61,6 & Regular & \\
\hline \multirow[t]{6}{*}{$10 / 11 / 2011$} & Lluvias & Paso de los anillos & 63,6 & Regular & \\
\hline & & Puente Córdoba & 61,9 & Regular & \\
\hline & & Finca la India & 63,4 & Regular & \\
\hline & & Finca el Confite & 62,1 & Regular & \\
\hline & & Muelle carbonífero & 59,1 & Regular & \\
\hline & & Desembocadura & 60,1 & Regular & \\
\hline \multirow[t]{6}{*}{$20 / 03 / 2012$} & Sequía & Paso de los anillos & 72,4 & Buena & \\
\hline & & Puente Córdoba & 72,2 & Buena & \\
\hline & & Finca la India & 71,2 & Buena & \\
\hline & & Finca el Confite & 70,3 & Buena & \\
\hline & & Muelle carbonífero & 67,9 & Regular & \\
\hline & & Desembocadura & 68,5 & Regular & \\
\hline
\end{tabular}


Tabla 3. Valores promedio de las variables físicas y químicas evaluadas en los seis sitios de estudio, durante los dos periodos climáticos. La numeración corresponde al nombre asignado a cada sitio de muestreo en la tabla 1. S: periodo de sequía, Ll: periodo de lluvias.

\begin{tabular}{|c|c|c|c|c|c|c|c|c|c|c|c|c|}
\hline \multirow{3}{*}{ Variable } & \multicolumn{12}{|c|}{ Sitio } \\
\hline & \multicolumn{2}{|c|}{1} & \multicolumn{2}{|c|}{2} & \multicolumn{2}{|c|}{3} & \multicolumn{2}{|c|}{4} & \multicolumn{2}{|c|}{5} & \multicolumn{2}{|c|}{6} \\
\hline & S & $\mathrm{Ll}$ & $\mathbf{S}$ & $\mathrm{Ll}$ & S & $\mathrm{Ll}$ & $\mathbf{S}$ & Ll & S & $\mathrm{Ll}$ & S & $\mathrm{Ll}$ \\
\hline $\begin{array}{l}\text { Alcalinidad } \\
(\mathrm{mg} / \mathrm{L} \mathrm{CaCO})\end{array}$ & 56,6 & 60,4 & 46,6 & 61,0 & 40,1 & 56,7 & 56,2 & 57,9 & 61,9 & 61,2 & 82,9 & 103,5 \\
\hline $\begin{array}{l}\text { Conductividad } \\
(\mu \mathrm{S} / \mathrm{cm})\end{array}$ & 89,7 & 112,1 & 66,0 & 98,8 & 100,1 & 97,7 & 125,4 & 104,4 & 120,4 & 100,0 & 120,5 & 100,3 \\
\hline $\begin{array}{l}\text { Color verdadero } \\
\text { (UPC) }\end{array}$ & 18,8 & 18,8 & 9,4 & 14,1 & 14,1 & 14,4 & 23,6 & 14,7 & 18,8 & 13,2 & 19,1 & 19,1 \\
\hline $\begin{array}{l}\text { Dureza total } \\
(\mathrm{mg} / \mathrm{L} \mathrm{CaCO})\end{array}$ & 61,9 & 60,1 & 43,7 & 45,8 & 46,6 & 48,6 & 47,3 & 53,4 & 44,0 & 55,7 & 55,8 & 67,0 \\
\hline $\mathrm{DQO}\left(\mathrm{mg} / \mathrm{L} \mathrm{O}_{2}\right)$ & 18,5 & 19,2 & 18,0 & 22,6 & 21,5 & 21,1 & 25,3 & 20,4 & 29,9 & 21,3 & 35,2 & 24,3 \\
\hline $\mathrm{DBO}\left(\mathrm{mg} / \mathrm{L} \mathrm{O}_{2}\right)$ & 2,9 & 7,4 & 3,4 & 7,8 & 4,3 & 8,3 & 4,0 & 7,4 & 7,1 & 16,0 & 6,9 & 13,3 \\
\hline $\begin{array}{l}\text { Oxígeno disuelto } \\
\left(\mathrm{mg} / \mathrm{L} \mathrm{O}_{2}\right)\end{array}$ & 5,6 & 6,0 & 5,6 & 6,2 & 5,7 & 7,0 & 4,9 & 6,1 & 5,3 & 6,1 & 5,2 & 5,8 \\
\hline pH (unidades) & 7,6 & 7,6 & 7,7 & 7,7 & 7,8 & 7,8 & 7,4 & 7,6 & 7,5 & 7,5 & 7,6 & 7,7 \\
\hline Hierro total (mg/L) & 0,1 & 0,3 & 0,1 & 0,3 & 0,1 & 0,3 & 0,1 & 0,3 & 0,1 & 0,4 & 0,1 & 0,3 \\
\hline Nitritos (mg/L NO & 0,1 & 0,1 & 0,0 & 0,1 & 0,0 & 0,1 & 0,1 & 0,1 & 0,2 & 0,1 & 0,2 & 0,1 \\
\hline Nitratos $\left(\mathrm{mg} / \mathrm{L} \mathrm{NO}_{3}\right)$ & 0,3 & 0,7 & 0,3 & 0,6 & 0,3 & 0,5 & 0,4 & 0,7 & 0,4 & 0,6 & 0,6 & 0,9 \\
\hline Fosfatos $\left(\mathrm{mg} / \mathrm{L} \mathrm{PO}_{4}\right)$ & 0,1 & 0,1 & 0,1 & 0,1 & 0,1 & 0,1 & 0,1 & 0,1 & 0,1 & 0,1 & 0,1 & 0,1 \\
\hline Sólidos totales (mg/L) & 94,5 & 355,0 & 104,7 & 409,0 & 105,2 & 280,0 & 113,4 & 397,0 & 137,4 & 287,0 & 146,4 & 309,0 \\
\hline $\begin{array}{l}\text { Sólidos suspendidos } \\
\text { (mg/L) }\end{array}$ & 13,1 & 128,8 & 16,5 & 159,7 & 17,2 & 80,7 & 22,3 & 136,7 & 21,3 & 54,1 & 20,0 & 105,1 \\
\hline $\begin{array}{l}\text { Sólidos disueltos } \\
\text { (mg/L) }\end{array}$ & 98,7 & 112,7 & 95,0 & 103,5 & 88,6 & 117,0 & 96,4 & 115,4 & 114,9 & 156,2 & 121,4 & 112,1 \\
\hline Turbiedad (UNT) & 1,8 & 27,2 & 1,5 & 27,7 & 1,5 & 29,6 & 1,6 & 31,3 & 3,1 & 33,0 & 3,6 & 28,9 \\
\hline Temperatura $\left({ }^{\circ} \mathrm{C}\right)$ & 26,1 & 23,4 & 26,5 & 23,8 & 27,0 & 24,6 & 26,3 & 26,4 & 26,3 & 25,8 & 25,9 & 25,3 \\
\hline $\begin{array}{l}\text { Coliformes totales } \\
\text { (NMP/100mL) }\end{array}$ & 4760 & 4845 & 3962 & 2470 & 4902 & 3800 & 5197 & 4085 & 6365 & 2898 & 6717 & 3943 \\
\hline $\begin{array}{l}\text { Coliformes fecales } \\
\text { (NMP/100mL) }\end{array}$ & 3012 & 4845 & 2974 & 4845 & 3021 & 4750 & 3078 & 4750 & 3373 & 4750 & 3553 & 4940 \\
\hline
\end{tabular}

A pesar de que en varios sectores de la parte baja del río Córdoba se utilizan fertilizantes, insecticidas y fungicidas, los valores de nitratos y fosfatos no indican contaminación del sistema. El aumento en el uso de fertilizantes con alto contenido en nitrógeno ha conducido a una mayor 108 como subterráneas, ya que la gran solubilidad de este compuesto implica que sea fácilmente liberado del suelo y arrastrado hasta los ríos por las aguas de escorrentía (Mendiguchía, 2005). Es probable que gran parte de los residuos de nitratos y fosfatos se concentren cerca de la desemboca el río Córdoba. De acuerdo con el criterio 
establecido para el diagnóstico y evaluación de la calidad del agua del río Córdoba, el agua correspondiente a los diferentes sitios de muestreo no es apta para el consumo humano porque presenta altos valores de coliformes fecales, de DBO y de turbiedad que sobrepasan los límites establecidos en la legislación colombiana y otros países, pero aptas para uso y protección de flora y fauna, según el Decreto 1076 de 2015.

Los valores del ICA-NSF que se registraron durante el segundo periodo seco, donde la mayoría de los sitios mostraron una "Buena" calidad del agua, pueden estar reflejando las condiciones normales del sistema, debido a que los muestreos del primer periodo de sequía se realizaron en un año Niña. Es probable que los resultados obtenidos el 11/08/2011 influyeran en los arrastres de material proveniente de las riberas del río y de aguas arriba, lo que provocó el aumento de algunas variables como la DBO, los nitratos y los coliformes fecales (Romero et al., 2011). En la parte baja del río Córdoba no se encontró evidencia de contaminación por la presencia significativa de residuos de compuestos organoclorados que afecten la salud humana y la supervivencia de especies de flora y fauna.

Finalmente, se debe considerar la valoración integral de los cuerpos de agua, de tal manera que se incluyan otros índices de calidad fisicoquímica (como el índice de contaminación mineral (ICOMI), contaminación por sólidos suspendidos (ICOSUS) y contaminación trófica (ICOTRO) y calidad (BMWP/Col y EPT). Con lo anterior, se propone que: 1) se puede conocer integralmente el estado de salud de estos sistemas (Forero-Céspedes et al., 2013), 2) se puedan hacer ajustes y validaciones de acuerdo a las condiciones locales y a los tipos de contaminantes que se evidencien en los sitios de interés (Zúñiga y Cardona, 2009; Montoya-M et al., 2011) y 3) los análisis respondan a los principales estresores identificados en cada localidad.

\section{Conclusiones}

La parte baja del cauce principal del río Córdoba mostró una calidad de agua categorizada como Regular y Buena, hecho que fue confirmado con las variables físicas y químicas que no presentaron valores que se excedieran de manera importante los rangos permisibles por la legislación nacional para fuentes de aguas naturales con uso recreativo y protección de la flora y la fauna acuática. Los resultados de coliformes fecales indican contaminación microbiológica del sistema en la medida que el cauce se acerca a la desembocadura del río, especialmente en los periodos de sequía. Las variables fisicoquímicas de agua se relacionaron con los periodos climáticos, pero no con variables espaciales del tramo. Estos resultados demuestran que la calidad del agua del río Córdoba en su tramo bajo no se considera como un riesgo para la biota del sistema y que los valores de las variables registradas en este trabajo fueron consistentes a los registrados en el río Manzanares por Romero (2011), cuyo río, al igual que el Córdoba, se ubica en la misma cara de la SNSM.

\section{Agradecimientos}

A Bienvenido Marín, Luis O. Duarte, Robert Melo Lora, Santiago González Rojas, Deimer Fontalvo y a Denis Alonso por la contribución que realizaron al documento. A Isaac Romero Borja, por el apoyo en los análisis de laboratorio. A María del Carmen Zúñiga, por la revisión del manuscrito. A los revisores anónimos por los comentarios para mejorar trabajo.

\section{Referencias}

APHA. 1989. Standards Methods for the Examination of Water and Wastewater, edition 20, American Public Health Association, EE.UU.

Ball, R., Church, R. 1980. Water Quality Indexing and Scoring. Journal of the Environmental Engineering División 106(4): 757-771.

Baron, J.S., LeRoy, N., Angermeier, P., Dahm, C., Gleick, P., Hairston, N., Jackson, R., Johnston, C., Richter, B. y Steinman, A. 2003. Ecosistemas de agua dulce sustentables. Tópicos en Ecología 10: 1-15.

Barrenechea, A. 2004. Tratamiento de agua para consumo humano. Plantas de filtración rápida. Manual I: Teoría, Tomo I. Centro Panamericano de Ingeniería Sanitaria y Ciencias del Ambiente, Lima, Perú.

Behar, R., Zúñiga, M. del C., Rojas, O. 1997. Análisis y valoración del Índice de Calidad de Agua (ICA) de la NSF: caos ríos Cali y Meléndez. Ingeniería y competitividad 1(1): 17-27.

Brown, R.M., McCleland, N.I., Deininger, R.A. y Tozer, R. 1970. A Water Quality Index - Do We Dare? Water and Sewage Works 117 (10): 339-343.

Bustamante, C.A., Naranjo, J.F. y Ahumada, J.M. 2014. Determinación de la calidad ecológica del río Tuluá Valle del Cauca. Revista Mutis 4(2): 35-43. 
Caballero, Y. 2007. Potencial Hidrológico y calidad de las aguas superficiales en la subcuenca del río Ochomogo. Tesis de maestría. Universidad Nacional Autónoma de Nicaragua.

Chapman, D.V. 1996. Water Quality Assessments: A guide to use Biota, Sediments and Water" Environmental Monitoring. Second Edition. UNESCO, WHO, and UNEP. E \& FN Spon, London UK.

Cadavid, G. y De Turbay, L. 1985. Manifestaciones culturales en el área Tairona. Informes antropológicos 1(5): 5-54.

Camacho, A., Giles, M., Ortegón, A., Palao, M., Serrano, B. y Velázquez, O. 2009. Técnicas para el Análisis Microbiológico de Alimentos. Segunda edición. Facultad de Química, Universidad Nacional Autónoma de México.

Castro, M., Almeida, J., Ferrer, J. y Díaz, D. 2014. Indicadores de la calidad del agua: evolución y tendencias a nivel global. Ingeniería solidaria 10(17): 111-124.

CODECI (Comité de Defensa Ciudadana). 1996. Notas ambientales. CODECI, Ciénaga, Magdalena.

CORFAS (Corporación Fondo de Apoyo de Empresas Asociativas). 1990. Plan de ordenación y Manejo de la cuenca del río Córdoba (parte alta). Santa Marta.

Díaz, M., González, M. y Pourrieux, J. 2018. Dirección de Recursos Hídricos de la Provincia de Tucumán. 2018 URL:http://www.recursoshidricos.gov.ar/webdrh/indexdrh. html.Consultado: 3 de febrero 2018.

Forero-Céspedes, A.M., Reinoso-Flórez, G., y Gutiérrez, C. 2013. Evaluación de la calidad del agua del río Opia (Tolima - Colombia) mediante macroinvertebrados acuáticos y parámetros fisicoquímicos. Caldasia 35(2): 371-387.

Hudson, N.W. 1997. Medición sobre el terreno de la erosión del suelo y de la escorrentía. Volumen 68. Organización de las Naciones Unidas para la Agricultura y la Alimentación, Roma.

INVEMAR. 2003. Programa holandés de asistencia para estudios de cambio climático, Colombia: Definición de la vulnerabilidad de los sistemas bio-geofísicos y socioeconómicos debido a un cambio en el nivel del mar en la zona costera colombiana (Caribe Continental, Caribe Insular y Pacífico) y medidas para su adaptación. Programa de Investigación para la Gestión Marina y Costera - GEZ, Santa Marta.

Jiménez, M.A. y Vélez, M.V. 2006. Análisis comparativo de indicadores de la calidad de agua superficial. Avances en 110 recursos hidráulicos 14: 53-69.
Mendiguchía, C. 2005. Utilización de ultratrazas de metales pesados como trazadores de los efectos antropogénicos producidos en ecosistemas acuáticos. Tesis doctoral. Universidad de Cádiz, Madrid, España.

MINAMBIENTE (Ministerio de Medio Ambiente y Recurso Naturales). 2005. Índice de Calidad del Agua General (ICA), Servicio Nacional de Estudios Territoriales, San Salvador.

Montoya-M, Y., Acosta, Y., y Zuluaga, E. 2011. Evolución de la calidad del agua en el río Negro y sus principales tributarios empleando como indicadores los índices ICA, el BMWP/Col y el ASPT. Caldasia 33(1): 193-210.

Murphy, J. y Riley, J.P. 1962. A modified single solution method for the determination of phosphate in natural waters. Analytica Chimica Acta 27: 31-36.

OMS (Organización Mundial de la Salud, US). 2006. Agua, saneamiento y salud: Enfermedades relacionadas con el agua_URL:_http://ho.int/wáter_saniation_health/diseases/ diseasefac/es/index.html. Consultado: 12 de diciembre 2010.

Pacheco, A. 2006. Diagnóstico de la Calidad del Agua en la Subcuenca del Rio Sedeño. Consejo del sistema veracruzano, México.

Parra, O. 2009. Gestión Integrada de Cuencas Hidrográficas. Centro de Ciencias Ambientales, Universidad de Concepción, Chile.

Pro-Sierra (Fundación Pro-Sierra Nevada de Santa Marta). 1991. Historia y Geografía Sierra Nevada de Santa Marta. Fundación Pro-Sierra Nevada de Santa Marta, Santafé de Bogotá, Colombia.

R Development Core Team. 2012. R: A language and environment for statistical computing. $\mathrm{R}$ Foundation for Statistical Computing, Viena, Austria. http://www.R-project. org/. Consultado: 15 de diciembre 2017.

Rivera, N.R., Encima, F., Muñoz-Pedreros, A. y Mejías, P. 2004. La Calidad de las Aguas en los Ríos Cautín e Imperial, Región-Chile. Información Tecnológica 15(5): 89-101.

Roldán, R. y Ramírez, J. 2008. Fundamentos de Limnología Neotropical. Segunda Edición. Universidad de Antioquia, Medellín.

Romero, I., Luna-Fontalvo, J.A. y Ponce, W. 2011. Calidad sanitaria de las fuentes hídricas de la cuenca baja del río Manzanares, Santa Marta, Colombia. Intropica 6: 51-62. 
Rúa-García, G. 2015. Macroinvertebrados acuáticos asociados a raíces de Eichhornia crassipes (mart) solms, en la ciénaga de Zapayán, Magdalena-Colombia. Intropica 10: 52-59.

Samboni-Ruíz, N.E., Carvajal-Escobar, Y. y Escobar, J.C. 2007. Revisión de parámetros fisicoquímicos como indicadores de calidad y contaminación del agua. Ingeniería e investigación 27(3): 172-181.

Toro, M., Robles, J., Avilés, J., Nuno, C., Vivas, S., Bonada, N., Prat, N., Alba, J., Casas, J., Guerrero, C., Jáimez-Cuéllar, P., Moreno, J., Moya, G., Suarez, M., Vidal - Abarca, M,. Álvarez, M. y Pardo, I. 2002. Calidad de las aguas de los ríos mediterráneos del proyecto GUADALMED. Características físico-químicas. Limnetica 21(3-4): 63-75.

Torres, J., Camacho, L. y Rodríguez, E. 2008. Marco de modelación de organismos patógenos en ríos de montaña. XVIII Seminario Nacional de Hidráulica e Hidrología. Sociedad Colombiana de Ingenieros. Bogotá, Colombia.
Torres, P., Hernán-Cruz, C., y Patiño, P. 2009. Índices de calidad de agua en fuentes superficiales utilizadas en la producción de agua para consumo humano. Una revisión crítica. Revista Ingenierías Universidad de Medellín 8(15): 79-94.

Valverde-Solis, A., Moreno-Tamayo, E., y Ortíz-Palacios, N.Y. 2015. Análisis de la calidad de varios cuerpos de aguas superciales en Bahía Solano utilizando índices de contaminación. Investigación, Biodiversidad y Desarrollo 34(1): 14-21.

Zúñiga, M. del C. y Cardona, W. 2009. Bioindicadores de calidad de agua y caudal ambiental. En: Cantera, J., Carvajal, J. y Castro, L.M. Compiladores. Caudal Ambiental: Conceptos, Experiencias y Desafíos. Universidad del Valle, Colombia.

Citar como: Fontalvo-Julio, F.A. y Tamaris-Turizo, C.E. 2018. Calidad del agua de la parte baja del río Córdoba (Magdalena, Colombia), usando el ICA-NSF. Intropica 13(2):101-111. DOI: http://dx.doi.org/10.21676/23897864.2510. 